\title{
Contrast-associated acute kidney injury is a myth: Yes
}

\author{
Stephan Ehrmann ${ }^{1,2,3^{*}}$ (D), Doron Aronson ${ }^{4,5}$ and Jeremiah S. Hinson ${ }^{6}$
}

(0) 2017 Springer-Verlag GmbH Germany, part of Springer Nature and ESICM

\section{Introduction}

Contrast medium (CM) administration is widely cited as a leading cause of hospital-acquired acute kidney injury (AKI) [1]. Concern over precipitation of AKI by CM is pervasive, and has influenced clinical decision-making related to diagnostic imaging and therapeutic interventions for more than half a century. So-called contrastinduced AKI (CI-AKI) is defined as an acute impairment in renal function occurring within 3 days of CM administration that is not attributable to any other etiology [1, 2]. Yet, nearly all studies establishing CI-AKI as a clinical entity were performed in the absence of control populations not exposed to CM. These studies assumed causality from association, and considered all cases of AKI in $\mathrm{CM}$-exposed patients as $\mathrm{CI}$-AKI, even when alternative explanations were obvious (Fig. 1) [3-5]. A growing body of evidence, derived from studies that include adequate control populations and discussed in more detail below, now suggests that risk for $\mathrm{AKI}$ attributable to $\mathrm{CM}$ administration is modest at most. Yet, outsized fear of CI-AKI persists.

\section{Clinical importance}

The distinction between a causal and associative relationship between CM and AKI is not purely academic. The prevailing belief that CM plays a central role in AKI has had major impacts over several decades, including promotion of potentially suboptimal clinical care at the patient level and subversion of a research agenda at the scientific level. Withholding CM on the basis of overestimated risk deprives individual patients of diagnostic studies and therapeutic interventions, many of which

\footnotetext{
*Correspondence: stephanehrmann@gmail.com

1 Médecine Intensive Réanimation, CHRU de Tours, Tours, France Full author information is available at the end of the article
}

For contrasting viewpoints, please go to https://doi.org/10.1007/s00134017-4970-2 and https://doi.org/10.1007/s00134-017-5015-6. convey substantial proven benefit $[6,7]$. There are scenarios in which CM administration is unavoidable, however, and the perceived risk of $\mathrm{CM}$ administration associated with such scenarios has driven intense interest in preventive measures for CI-AKI. Indeed, an entire field of investigation aimed at their identification and evaluation has been established. An enormous amount of funding has been allocated to this line of research and a very large number of patients have been exposed to experimental interventions including $\mathrm{N}$-acetylcysteine, sodium bicarbonate, fluid loading, statins, vitamin $C$, vasoactive medications, theophylline, preconditioning ischemia, and renal replacement therapy-none of which are devoid of side effects [8]. The preponderance of negative trials testing similar preventive strategies for $\mathrm{CI}-\mathrm{AKI}$ is in itself a strong argument against a causative relationship between $\mathrm{CM}$ and $\mathrm{AKI}$, and even raises potential ethical concerns for enrollment of patients in such studies. Interestingly, all three authors of this editorial, as initial believers in CI-AKI, planned or initiated interventional trials aimed at its prevention in their respective fields of emergency medicine, critical care, and cardiology. In doing so, each abandoned their efforts after independently concluding, in the light of the available literature, that the role of $\mathrm{CM}$ in AKI has been vastly overestimated.

\section{Intravenous contrast media and acute kidney injury in the general population}

Studies performed in unselected populations with controls do not support a meaningful role for intravenous $\mathrm{CM}$ in causing AKI. Meta-analysis of 13 studies in which incidence of AKI was directly compared between patients who were and were not exposed to CM failed to demonstrate any increased risk of AKI, dialysis, or death associated with CM [9]. This is supported by a recent nationwide analysis of administrative data for almost 6 million patients in the USA, where rates of AKI were

\section{Springer}




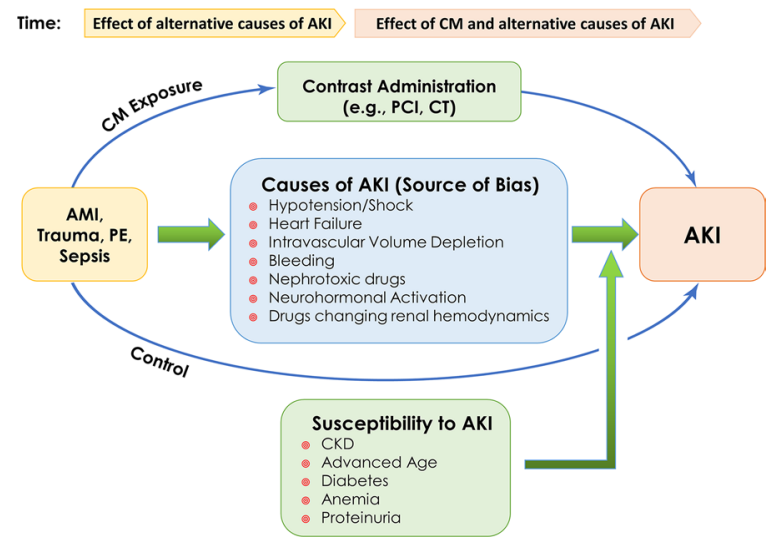

Fig. 1 Diagram showing the importance of negative controls in evaluating the causal relationship between contrast media (CM) exposure and acute kidney injury (AKI). Regardless of the decision to use CM, acute illness (acute myocardial infraction, sepsis, trauma, etc.) produces a set of alternative conditions that can independently initiate the process of AKI prior to, during, and after the administration of $\mathrm{CM}$. Note that the potential bias introduced by other causes of AKI increases with increasing severity of the acute illness. The essential purpose of the negative control group is to reproduce a condition that does not include the hypothesized causal mechanism but is very likely to involve the same sources of bias that are often present in the original association. If contaminants (source of bias) were responsible for the effect of the $C M$, they should exert their effect even when CM is not present. A control group allows us to estimate the true effect of $\mathrm{CM}$ exposure given the background of multiple confounders. In controlled studies, the outcome (AKI) is encountered without exposure to $\mathrm{CM}$, demonstrating the existence of a confounding bias. AMl acute myocardial infarction, $\mathrm{PE}$ pulmonary embolism, $\mathrm{PCl}$ percutaneous coronary intervention, CT computed tomography, AKI acute kidney injury, CM contrast media, CKD chronic kidney disease

nearly identical in patients who did and did not receive CM [10]. Direct comparison of populations, as performed in these studies, is confounded by selection bias associated with the decision to administer or withhold CM. Several subsequent studies have utilized propensity matching analysis to minimize this bias. None of these studies identified an independent association between intravenous $\mathrm{CM}$ administration and risk for AKI, dialysis, or death in the general population [11-13]. While one group did report increased risk of AKI associated with $\mathrm{CM}$ administration in the less than $5 \%$ of patients with an estimated glomerular filtration rate below $30 \mathrm{~mL} /$ $\mathrm{min} / 1.73 \mathrm{~m}^{2}$, two others did not [11-13].

\section{Contrast media and acute kidney injury in the critically ill}

Critically ill patients have long been considered a population at particularly elevated risk of CI-AKI [14]. A recent systematic review revealed that this belief has been driven by a large body of uncontrolled data [15]. Meta-analysis of three propensity-matched controlled studies showed a lack of significant difference in AKI incidence among 560 critically ill patients who were exposed or unexposed to CM [15]. Those data were reinforced by a large monocentric database analysis showing a lack of significant association between CM and AKI, renal replacement therapy, and mortality among 6877 propensity-matched intensive care unit patients [16]. These findings have important clinical implications, as the utility of $\mathrm{CM}$ for diagnosis and treatment of immediately life-threatening conditions may be even greater among critically ill patients than in the general population.

\section{Contrast media and acute kidney injury in patients undergoing percutaneous intervention}

The reported incidence of CI-AKI following percutaneous intervention (PCI) ranges from $1-2 \%$ in patients undergoing elective PCI to $10-20 \%$ in patients undergoing PCI for ST-elevation myocardial infarction (STEMI) $[1,3,4,7]$. While attributed to CM exposure, the marked increase in AKI rates in these patients more likely results from associated conditions including hypotension, hemodynamic instability/cardiogenic shock, acute heart failure, hemorrhage, and initiation of medications that alter renal hemodynamics (Fig. 1). Indeed, multiple retrospective controlled studies in patients undergoing PCI were unable to show a meaningful adverse effect of $\mathrm{CM}$ exposure $[6,7,10]$. Further, a study performed in a large inpatient sample that controlled for comorbidity and acuity of illness found that patients with acute coronary syndromes $(n=1,251,812)$ who received CM experienced an unexpectedly lower rate of AKI as compared to patients who were not exposed to contrast $(6.4 \%$ versus $17.4 \%$ ) [10]. Finally, a propensity-matched cohort study of patients with non-STEMI reported one additional episode of AKI for every 62 participants treated with an early invasive approach instead of a conservative approach, with similar risks of dialysis or long-term risk of end-stage renal disease, but better long-term survival with the invasive approach [6] and in a propensitymatched cohort study of patients with STEMI, the risk of AKI was similar with and without CM exposure [7].

\section{Conclusion}

Mythology and science share a common purpose: to provide meaning to and understanding of phenomena that are readily observed but not easily explained. The two disciplines diverge dramatically in process, however, with the prior relying on imagination and conjecture and the latter on a rigorous method that includes observation followed by hypothesis formulation, testing through structured experimentation, and finally rejection or acceptance. In keeping with this, and the data discussed above, we support the clause under debate: 
contrast-induced acute kidney injury is a myth. The concept of CI-AKI arose from observation and assumed causality, and despite a lack of rigorous hypothesis testing, has driven clinical practice for more than five decades. While a randomized controlled trial has not been conducted, there is now substantial evidence suggesting that CM contributes minimally, if at all, to the development of AKI $[6,7,9,11,13,15-17]$. Despite the existence of such evidence, a pervasive preoccupation with what should be referred to as contrast-associated AKI (CA-AKI) persists in clinical practice, medical texts, and even among clinical researchers. There are many well-established risk factors for AKI (Fig. 1), many of which have clear causal relationships with AKI and are readily modifiable, yet are often ignored as episodes of AKI are attributed to CM. Disproportionate focus on CM also results in misappropriation of critical human and monetary capital that could be more effectively harnessed to reduce AKI-associated harms.

\begin{abstract}
Author details
${ }^{1}$ Médecine Intensive Réanimation, CHRU de Tours, Tours, France. ${ }^{2}$ Faculté de médecine, Université François Rabelais, Tours, France. ${ }^{3}$ CRICS-Triggersep Network, Tours, France. ${ }^{4}$ Department of Cardiology, Rambam Medical Center, Haifa, Israel. ${ }^{5}$ Ruth and Bruce Rappaport Faculty of Medicine, Technion, Israel Institute of Technology, Haifa, Israel. ${ }^{6}$ Department of Emergency Medicine, Johns Hopkins University School of Medicine, Baltimore, MD, USA.
\end{abstract}

\section{Compliance with ethical standards}

\section{Conflicts of interest}

None of the authors has a conflict of interest related to the present manuscript.

Received: 19 September 2017 Accepted: 23 September 2017 Published online: 14 December 2017

\section{References}

1. Mehran R, Nikolsky E (2006) Contrast-induced nephropathy: definition, epidemiology, and patients at risk. Kidney Int Supp1:S11-S15

2. Persson PB, Hansell P, Liss P (2005) Pathophysiology of contrast mediuminduced nephropathy. Kidney Int 68:14-22

3. Marenzi G, Assanelli E, Campodonico J, Lauri G, Marana I, De Metrio M, Moltrasio M, Grazi M, Rubino M, Veglia F, Fabbiocchi F, Bartorelli AL (2009) Contrast volume during primary percutaneous coronary intervention and subsequent contrast-induced nephropathy and mortality. Ann Intern Med 150:170-177

4. Narula A, Mehran R, Weisz G, Dangas GD, Yu J, Genereux P, Nikolsky E, Brener SJ, Witzenbichler B, Guagliumi G, Clark AE, Fahy M, Xu K, Brodie
BR, Stone GW (2014) Contrast-induced acute kidney injury after primary percutaneous coronary intervention: results from the HORIZONS-AMI substudy. Eur Heart J 35:1533-1540

5. Lipsitch M, Tchetgen Tchetgen E, Cohen T (2010) Negative controls: a tool for detecting confounding and bias in observational studies. Epidemiology 21:383-388

6. James MT, Tonelli M, Ghali WA, Knudtson ML, Faris P, Manns BJ, Pannu N, Galbraith PD, Hemmelgarn BR (2013) Renal outcomes associated with invasive versus conservative management of acute coronary syndrome: propensity matched cohort study. BMJ 347:f4151

7. Caspi O, Habib M, Cohen Y, Kerner A, Roguin A, Abergel E, Boulos M, Kapeliovich MR, Beyar R, Nikolsky E, Aronson D (2017) Acute kidney injury after primary angioplasty: is contrast-induced nephropathy the culprit? J Am Heart Assoc 6:e005715

8. Fishman EK, Reddan D (2008) What are radiologists doing to prevent contrast-induced nephropathy (CIN) compared with measures supported by current evidence? A survey of European radiologists on CIN associated with computed tomography. Acta Radiol 49:310-320

9. McDonald JS, McDonald RJ, Comin J, Williamson EE, Katzberg RW, Murad $\mathrm{MH}$, Kallmes DF (2013) Frequency of acute kidney injury following intravenous contrast medium administration: a systematic review and meta-analysis. Radiology 267:119-128

10. Wilhelm-Leen E, Montez-Rath ME, Chertow G (2017) Estimating the risk of radiocontrast-associated nephropathy. J Am Soc Nephrol 28:653-659

11. McDonald JS, McDonald RJ, Carter RE, Katzberg RW, Kallmes DF, Williamson EE (2014) Risk of intravenous contrast material-mediated acute kidney injury: a propensity score-matched study stratified by baselineestimated glomerular filtration rate. Radiology 271:65-73

12. Davenport MS, Khalatbari S, Dillman JR, Cohan RH, Caoili EM, Ellis JH (2013) Contrast material-induced nephrotoxicity and intravenous lowosmolality iodinated contrast material. Radiology 267:94-105

13. Hinson JS, Ehmann MR, Fine DM, Fishman EK, Toerper MF, Rothman RE, Klein EY (2017) Risk of acute kidney injury after intravenous contrast media administration. Ann Emerg Med 69(577-586):e4

14. Lakhal K, Ehrmann S, Chaari A, Laissy JP, Régnier B, Wolff M, Pajot O (2011) Acute kidney injury network definition of contrast-induced nephropathy in the critically ill: incidence and outcome. J Crit Care 26:593-599

15. Ehrmann S, Quartin A, Hobbs BP, Robert-Edan V, Cely C, Bell C, Lyons G, Pham T, Schein R, Geng Y, Lakhal K, Ng CS (2017) Contrast-associated acute kidney injury in the critically ill: systematic review and Bayesian meta-analysis. Intensive Care Med 43:1017-1026

16. McDonald JS, McDonald RJ, Williamson EE, Kallmes DF, Kashani K (2017) Post-contrast acute kidney injury in intensive care unit patients: a propensity score-adjusted study. Intensive Care Med 43:774-784

17. Brinjikji W, Demchuk AM, Murad MH, Rabinstein AA, McDonald RJ, McDonald JS, Kallmes DF (2017) Neurons over nephrons: systematic review and meta-analysis of contrast-induced nephropathy in patients with acute stroke. Stroke 48:1862-1868 